\title{
Symmetries and Reduction for Multi-Agent Control
}

\author{
Ashley Nettleman ${ }^{1}$ and Bill Goodwine ${ }^{2}$
}

\begin{abstract}
This paper computes all the continuous pointtransformation symmetries for a planar multi-agent dynamical control system. The relative configuration among the agents is often the most important aspect of this problem, and this paper presents a method to "factor out" the location and orientation on the plane of a multi-agent formation problem. This is useful in the case where only the relative positioning of the agents is of concern. Furthermore, in the case where the location of the formation is still important, it provides a basis for splitting the problem into the "formation" part and the "location of the formation" part. The method to compute the symmetries and determine the reduced coordinates is well-established. The contributions of this paper are 1) computing the symmetries for a multi-agent system, 2) highlighting the utility of the reduction for the formation control application such as formation stability and 3) analyzing the symmetries to determine a relatively broad class of systems which have the same symmetries and hence identical coordinates for reduction. Although it has been addressed in various ways, essentially all methods to determine the stability properties of the formation control problem need the dynamics to be formulated on a reduced, relative space. Hence, stability (and stability-like notions such as that which follow from LaSalle's Principle) analyses will be the main beneficiary of this work.
\end{abstract}

\section{BACKGROUND}

Consider a planar formation control problem where the control law for each agent depends only on its relative position to the other agents as well as its velocity (but not its absolute position). In such a case it makes sense that if we compute the solution for the system for a given set of initial positions and then compute the solution where all the initial positions are subjected to the same Euclidean transformation, the solution for the transformed initial conditions would be the same as the solution for the original system, but simply subjected to the same transformation. This is illustrated in Figure 1 for an example considered in detail subsequently.

The contributions of this paper are 1) an explicit computation of the symmetries for a multi-agent system, 2) the construction of a coordinate transformation which aligns the coordinates with the action of the symmetries (which reduces of the dynamics to the essential relative dynamics), 3 ) an analysis of the resulting system and symmetries which provides insight into the very broad class of systems to which the reduction can apply and 4) an analysis of the transformed dynamics of the system which make it apparent that the dynamics in such transformed coordinates are the easiest

*The support of the US National Science Foundation under the CPS Large Grant No. CNS-1035655 is gratefully acknowledged.

${ }^{1}$ Both authors are with the Department of Aerospace \& Mechanical Engineering, University of Notre Dame, Notre Dame, Indiana 46556 USA, akulczycend.edu

2 billdcontrols. ame.nd.edu

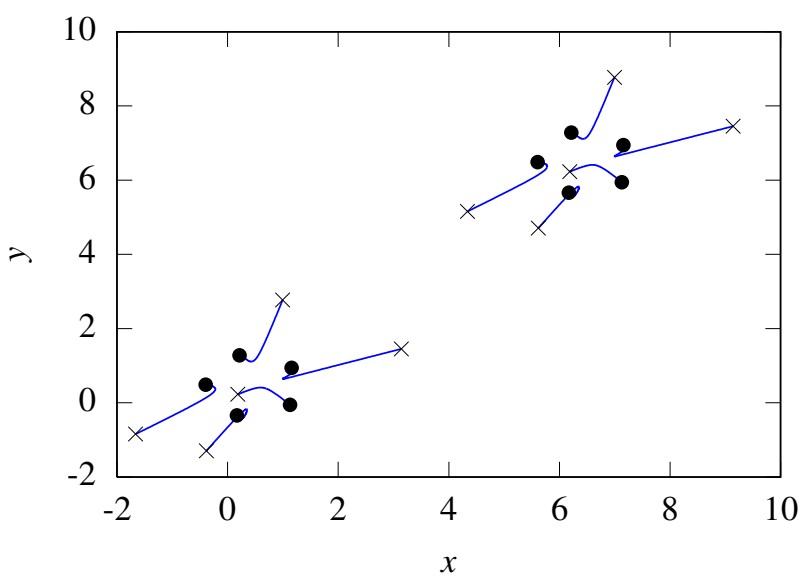

Fig. 1. Multi-agent system with dynamics invariant under Euclidean transformation. The $\times \mathrm{s}$ are the initial conditions and the circles are the final positions.

ones in which to consider stability-like notions in formation control.

The application of symmetry analysis to differential equations is well-established (see [1], [2]) with much attention particularly in mechanics [3]-[8]. One closely-related publication is [9], which considers the reduction problem specifically for two rigid bodies in $S E(3)$ and discusses the $N$ rigid body case. However, in contrast to this paper, it considers the problem specifically in the context of Hamiltonian mechanics. We take a more "direct computation" approach to determining and using the symmetries without appeal to mechanics, which provides another dimension to our contribution. Formation control and multi-agent control of cyber-physical systems, of course, has a vast literature and representative references include [10]-[24]. A publication closely-related in spirit is [25] dealing with formation control decomposing the dynamics of a group of agents into the product of a group configuration (configuration in space) and a shape manifold.

A prototypical motivation to consider throughout this paper is the question of whether a formation is stable (in some sense). This is a complicated question because even if the dynamics of the problem are such that the while the relative configuration among the robots converges to a desired final value, it may be the case that the final formation has an overall constant translational and/or rotational velocity. This is problematic for tests of stability notions because 1) there clearly is a set of configurations which are the formation, and hence the nonlinear analysis tool to use is LaSalle's Principle and 2) LaSalle's Principle requires an invariant compact set, which is problematic when the final formation has a non-zero 


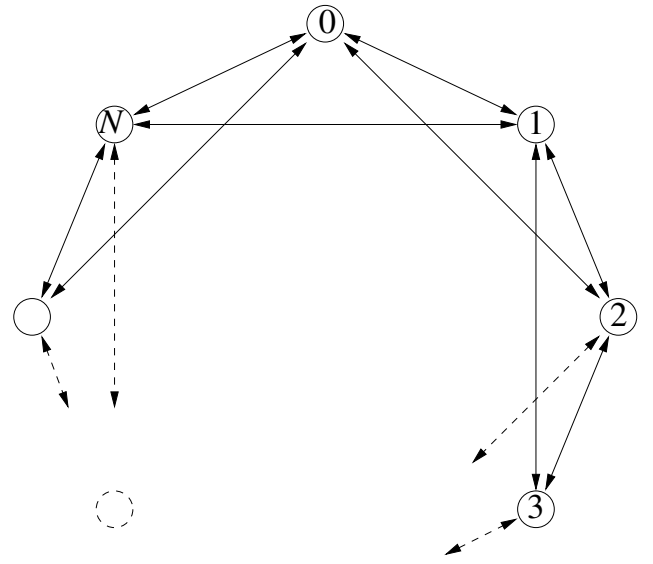

Fig. 2. System topology for Example 1.

steady-state velocity. Such difficulties are typically addressed in a case-specific manner, for example using notions such as moving center of mass coordinates in a formation [26] or in the consensus context, by defining a "disagreement functions" [27]-[29]. These are, in effect, going "part way" to exploit the translational symmetries present in the system, and the contribution of this paper is that we explicitly do so.

The example throughout this paper is the following.

Example 1: Consider the system illustrated in Figure 2 where the goal formation is a regular $N$-polygon. Each agent is a point mass with second-order dynamics, and the control law (formation force) for each agent is from a quadratic potential based on the deviation from the desired distance from its neighbors. The set of neighbors considered by each robot is four other robots, its two nearest neighbors in each direction. Hence, the nonlinear equations of motion are

$$
\begin{aligned}
& \ddot{x}_{i}=-\dot{x}_{i}+\sum_{j \in \mathscr{N}_{i}}\left(x_{i}-x_{j}\right)\left(\left(x_{i}-x_{j}\right)^{2}+\left(y_{i}-y_{j}\right)^{2}-\hat{d}_{i j}\right) \\
& \ddot{y}_{i}=-\dot{y}_{i}+\sum_{j \in \mathscr{N}_{i}}\left(y_{i}-y_{j}\right)\left(\left(x_{i}-x_{j}\right)^{2}+\left(y_{i}-y_{j}\right)^{2}-\hat{d}_{i j}\right)
\end{aligned}
$$

where $\hat{d}_{i j}$ is the desired distance between robots $i$ and $j$,

$$
\hat{d}_{i j}=\left\{\begin{array}{ll}
1, & |i-j|=1 \\
\frac{\sin 2 \pi / N}{\sin \pi / N}, & |i-j|=2
\end{array} .\right.
$$

It will be clear that the results in this paper will apply regardless of which of the other agents are an agent's neighbors.

\section{SYMMETRIES OF A SYSTEM OF SECOND-ORDER EQUATIONS}

Throughout this section we generally follow the development and notation from [2]. A system of $m$ second order differential equations

$$
\begin{gathered}
\ddot{q}^{1}=\omega^{1}\left(q^{1}, \ldots, q^{m}, \dot{q}^{1}, \ldots, \dot{q}^{m}\right) \\
\vdots \\
\ddot{q}^{m}=\omega^{m}\left(q^{1}, \ldots, q^{m}, \dot{q}^{1}, \ldots, \dot{q}^{m}\right),
\end{gathered}
$$

can be written as a partial differential equation $A f=0$, where

$$
A=\frac{\partial}{\partial t}+\dot{q}^{1} \frac{\partial}{\partial q^{1}}+\omega^{1} \frac{\partial}{\partial \dot{q}^{1}}+\cdots+\dot{q}^{m} \frac{\partial}{\partial q^{m}}+\omega^{m} \frac{\partial}{\partial \dot{q}^{m}} .
$$

Typically symmetry analysis is easier using a linear operator than the full, typically nonlinear, symmetry transformation. Such a linear operator can be considered the "velocity" of the transformation (in this context called a generator), and if a system of equations is invariant in that direction at all points, then it will be invariant under the full transformation.

If our goal is to determine all the symmetries, then we express the generator in an general form and apply it to the system of interest to determine the unknown terms in the generator. In this case the general form for a symmetry expressed as a generator, $X$, of $m$ second order differential equations is the vector field

$$
\begin{aligned}
X= & \xi\left(t, q^{1}, \ldots, q^{m}\right) \frac{\partial}{\partial t}+\eta^{1}\left(t, q^{1}, \ldots, q^{m}\right) \frac{\partial}{\partial q^{1}}+\cdots \\
& +\eta^{m}\left(t, q^{1}, \ldots, q^{m}\right) \frac{\partial}{\partial q^{m}} .
\end{aligned}
$$

Invariance under the action of the generator is expressed by the Lie commutator $[X, A]=\lambda A$, which, for a system of second-order equations, may be expressed as

$$
\begin{aligned}
x i \omega_{, t}^{a}+ & \eta^{b} \omega_{, b}^{a}+\left(\eta_{, t}^{b}+\dot{q}^{c} \eta_{, c}^{b}-\dot{q}^{b} \xi_{, t}-\dot{q}^{b} \dot{q}^{c} \xi_{, c}\right) \frac{\partial \omega^{a}}{\partial \dot{q}^{b}} \\
+ & 2 \omega^{a}\left(\xi_{, t}+\dot{q}^{b} \xi_{, b}\right)+\omega^{b}\left(\dot{q}^{a} \xi_{, b}-\eta_{, b}^{a}\right)+\dot{q}^{a} \dot{q}^{b} \dot{q}^{c} \xi_{, b c} \\
& +2 \dot{q}^{a} \dot{q}^{c} \xi_{, t c}-\dot{q}^{c} \dot{q}^{b} \eta_{, b c}^{a}+\dot{q}^{a} \xi_{, t t}-2 \dot{q}^{b} \eta_{, t b}^{a}-\eta_{, t t}^{a}=0,
\end{aligned}
$$

with $(a, b, c=1, \ldots, m)$ and, as conventional, $\eta_{, a}$ represents partial differentiation with respect to $q_{a}$ and we adopt the summation convention (so $a$ is the free index). Because $\xi$ and $\eta^{a}$ are functions of $t$ and $q^{a}$ and not $\dot{q}^{a}$, we are able to arrange the equations in the order of the $\dot{q}^{a}$ terms.

Returning to the example, we may write

$$
\ddot{q}^{i}=\omega^{i}=-\dot{q}^{i}-\sum_{j \in \mathscr{N}_{i}}\left(q^{i}-q^{l}\right)\left(\left(q^{i}-q^{l}\right)^{2}+\left(q^{k}-q^{m}\right)^{2}-\hat{d}_{j}^{i}\right)
$$

where $i \in\{0, \ldots, 2 N\}, k=i-(-1)^{i}, l=i+2 j$ $(\bmod 2 N), m=k+2 j(\bmod 2 N)$ and $q^{i}$ is either $x$ or $y$ depending on whether the index is odd or even. Note that the system does not depend explicitly on time and the $\dot{q}^{a}$ terms do not appear in the input force from the formation potential, so $\ddot{q}^{i}=\omega^{i}=-\dot{q}^{i}-g^{i}$ with $g^{a}\left(q^{1}, \ldots, q^{2 N}\right)$ only a function of the configuration and not velocities.

The $m=2 N$ equations that define the symmetry are

$$
\begin{array}{r}
\eta^{b}\left(-g^{a}\right)_{, b}+\left(\eta_{, t}^{b}+\dot{q}^{c} \eta_{, c}^{b}-\dot{q}^{b} \xi_{, t}-\dot{q}^{b} \dot{q}^{c} \xi_{, c}\right)(-1) \delta_{a b} \\
+2\left(-\dot{q}^{a}-g^{a}\right)\left(\xi_{, t}+\dot{q}^{b} \xi_{, b}\right)+\left(-\dot{q}^{b}-g^{b}\right)\left(\dot{q}^{a} \xi_{, b}-\eta_{, b}^{a}\right) \\
+\dot{q}^{a} \dot{q}^{b} \dot{q}^{c} \xi_{, b c}+2 \dot{q}^{a} \dot{q}^{c} \xi_{, t c}-\dot{q}^{c} \dot{q}^{b} \eta_{, b c}^{a}+\dot{q}^{a} \xi_{, t t}-2 \dot{q}^{b} \eta_{, t b}^{a} \\
-\eta_{, t t}^{a}=0,
\end{array}
$$


with $\delta_{a b}=1$ if $a=b$ and 0 otherwise. Rearranging in decreasing powers of $\dot{q}^{a}$ gives

$$
\begin{aligned}
& \dot{q}^{a} \dot{q}^{b} \dot{q}^{c} \xi_{, b c}+\dot{q}^{b} \dot{q}^{c} \xi_{, c} \delta_{a b}-3 \dot{q}^{a} \dot{q}^{b} \xi_{, b}+2 \dot{q}^{a} \dot{q}^{c} \xi_{, t c}-\dot{q}^{c} \dot{q}^{b} \eta_{, b c}^{a} \\
& -\dot{q}^{c} \eta_{, c}^{b} \delta_{a b}+\dot{q}^{b} \xi_{, t} \delta_{a b}-2 \dot{q}^{a} \xi_{, t}-2 \dot{q}^{b} g^{a} \xi_{, b}+\dot{q}^{b} \eta_{, b}^{a}-\dot{q}^{a} g^{b} \xi_{, b} \\
+ & \dot{q}^{a} \xi_{, t t}-2 \dot{q}^{b} \eta_{, t b}^{a}-\eta^{b} g_{, b}^{a}-\eta_{, t}^{b} \delta_{a b}-2 g^{a} \xi_{, t}+g^{b} \eta_{, b}^{a}-\eta_{, t t}^{a}=0 .
\end{aligned}
$$

Because the $\dot{q}^{a}$ are independent for different orders, the coefficient of each term much be zero. Thus $\dot{q}^{a} \dot{q}^{b} \dot{q}^{c} \xi_{, b c}=0$ gives that

$$
\xi=\alpha^{0}(t)+\alpha^{1}(t) q^{1}+\cdots+\alpha^{2 N}(t) q^{2 N},
$$

which gives

$$
\begin{aligned}
\xi_{, t} & =\dot{\alpha}^{0}(t)+\dot{\alpha}^{1}(t) q^{1}+\cdots+\dot{\alpha}^{2 N}(t) q^{2 N} \\
\xi_{, b} & =\alpha^{b}(t) \\
\xi_{, t b} & =\dot{\alpha}^{b}(t) .
\end{aligned}
$$

The symmetry condition equations are now

$$
\begin{gathered}
\dot{q}^{b} \dot{q}^{c} \alpha^{c}(t) \delta_{a b}-3 \dot{q}^{a} \dot{q}^{b} \alpha^{b}(t)+2 \dot{q}^{a} \dot{q}^{c} \dot{\alpha}^{c}(t)-\dot{q}^{c} \dot{q}^{b} \eta_{, b c}^{a} \\
-\dot{q}^{c} \eta_{, c}^{b} \delta_{a b}+\dot{q}^{b} \delta_{a b}\left(\dot{\alpha}^{0}(t)+\dot{\alpha}^{c}(t) q^{c}\right)-2 \dot{q}^{a}\left(\dot{\alpha}^{0}(t)+\dot{\alpha}^{c}(t) q^{c}\right) \\
-2 \dot{q}^{b} g^{a} \alpha^{b}(t)+\dot{q}^{b} \eta_{, b}^{a}-\dot{q}^{a} g^{b} \alpha^{b}(t)+\dot{q}^{a}\left(\ddot{\alpha}^{0}(t)+\ddot{\alpha}^{c}(t) q^{c}\right) \\
-2 \dot{q}^{b} \eta_{, t b}^{a}-\eta^{b} g_{, b}^{a}-\eta_{, t}^{b} \delta_{a b}-2 g^{a}\left(\dot{\alpha}^{0}(t)+\dot{\alpha}^{c}(t) q^{c}\right)+g^{b} \eta_{, b}^{a} \\
-\eta_{, t t}^{a}=0,
\end{gathered}
$$

Proceeding to the next lower-order term gives

$$
\dot{q}^{b} \dot{q}^{c} \alpha^{c}(t) \delta_{a b}-3 \dot{q}^{a} \dot{q}^{b} \alpha^{b}(t)+2 \dot{q}^{a} \dot{q}^{c} \dot{\alpha}^{c}(t)-\dot{q}^{c} \dot{q}^{b} \eta_{, b c}^{a}=0
$$

which simplifies to

$$
2 \dot{q}^{a} \dot{q}^{b}\left(\dot{\alpha}^{b}(t)-\alpha^{b}(t)\right)-\dot{q}^{c} \dot{q}^{b} \eta_{, b c}^{a}=0 .
$$

Recall that $a$ designates which symmetry condition equation while $b$ and $c$ are summation indices. This gives that

$$
\begin{aligned}
2\left(\dot{\alpha}^{b}(t)-\alpha^{b}(t)\right) & =\eta_{, b a}^{a}, \\
\eta_{, b c}^{a} & =0 \text { when } c \neq a .
\end{aligned}
$$

Since partial derivatives commute,

$$
\begin{aligned}
\eta_{, b c}^{a} & =0, \\
\eta^{a} & =\beta^{a}(t)+\gamma_{b}^{a}(t) q^{b} \\
2\left(\dot{\alpha}^{b}(t)-\alpha^{b}(t)\right) & =0 \\
\dot{\alpha}^{b}(t) & =\alpha^{b}(t), \\
\alpha^{b}(t) & =\alpha^{b} \exp (t),
\end{aligned}
$$

where $\alpha^{b}$ is a scalar.

The symmetry condition equations are now

$$
\begin{aligned}
& -\dot{q}^{a} \dot{\alpha}^{0}(t)+\dot{q}^{a} \ddot{\alpha}^{0}(t)-2 \dot{q}^{b} \dot{\gamma}_{b}^{a}(t)-2 \dot{q}^{b} g^{a} \alpha^{b} \exp (t) \\
- & \dot{q}^{a} g^{b} \alpha^{b} \exp (t)-g_{, b}^{a} \beta^{b}(t)-g_{, b}^{a} \gamma_{c}^{b}(t) q^{c}-\dot{\gamma}_{b}^{a}(t) q^{b}-2 g^{a} \dot{\alpha}^{0}(t) \\
- & 2 g^{a} \alpha^{b} \exp (t) q^{b}+g^{b} \gamma_{b}^{a}(t)-\ddot{\gamma}_{b}^{a}(t) q^{b}-\dot{\beta}^{a}(t)-\ddot{\beta}^{a}(t)=0 .
\end{aligned}
$$

In the general case, one would continue with the $\dot{q}^{i}$ terms, however, note that the purely time based terms are quite simple to solve. The equation to solve is $\dot{\beta}^{a}(t)+\ddot{\beta}^{a}(t)=$
0 , which gives $\beta^{a}(t)=\beta_{1}^{a}+\beta_{2}^{a} \exp (-t)$, where $\beta_{1}^{a}$ and $\beta_{2}^{a}$ are constants. The updated expression for $\eta^{a}$ is $\eta^{a}=\beta_{1}^{a}+$ $\beta_{2}^{a} \exp (-t)+\gamma_{b}^{a}(t) q^{b}$.

The symmetry condition equations are now

$$
\begin{array}{r}
-\dot{q}^{a} \dot{\alpha}^{0}(t)+\dot{q}^{a} \ddot{\alpha}^{0}(t)-2 \dot{q}^{b} \dot{\gamma}_{b}^{a}(t)-2 \dot{q}^{b} g^{a} \alpha^{b} \exp (t) \\
-\dot{q}^{a} g^{b} \alpha^{b} \exp (t)-g_{, b}^{a}\left(\beta_{1}^{b}+\beta_{2}^{b} \exp (-t)\right)-g_{, b}^{a} \gamma_{c}^{b}(t) q^{c} \\
-\dot{\gamma}_{b}^{a}(t) q^{b}-2 g^{a} \dot{\alpha}^{0}(t)-2 g^{a} \alpha^{b} \exp (t) q^{b}+g^{b} \gamma_{b}^{a}(t) \\
-\ddot{\gamma}_{b}^{a}(t) q^{b}=0 .
\end{array}
$$

At this point the formation force needs to be substituted for $g^{a}$ to solve the $\dot{q}^{a}$ and $q^{a}$ equations, which will be omitted due to space limitations. The final result is the collection of expressions

$$
\begin{aligned}
& X=\xi \frac{\partial}{\partial t}+\eta^{a} \frac{\partial}{\partial q^{a}} \\
& \xi=\alpha^{0} \\
& \bar{\eta}=\overline{\beta(t)}+\bar{\Gamma} \cdot \bar{q}=\left(\eta^{1}, \ldots, \eta^{2 N}\right)^{T}, \\
& \bar{q}=\left(q^{1}, \ldots, q^{2 N}\right)^{T}, \quad \overline{\beta(t)}=\left(\beta^{1}(t), \ldots, \beta^{2 N}(t)\right)^{T}, \\
& \beta^{i}(t)=\left\{\begin{array}{ll}
\beta_{1}^{1}+\beta_{2}^{1} \exp (-t) & \text { for } i \text { odd } \\
\beta_{1}^{2}+\beta_{2}^{2} \exp (-t) & \text { for } i \text { even }
\end{array},\right. \\
& \bar{\Gamma}=\left(\begin{array}{cccc}
B & C & \cdots & C \\
C & B & & \vdots \\
\vdots & & \ddots & C \\
C & \ldots & C & B
\end{array}\right), \\
& B=\left(\begin{array}{ll}
\gamma_{1}^{1} & \gamma_{2}^{1} \\
\gamma_{1}^{2} & \gamma_{2}^{2}
\end{array}\right), \quad C=\left(\begin{array}{cc}
\gamma_{1}^{1} & \gamma_{4}^{1} \\
\gamma_{2}^{1}-\gamma_{4}^{1}+\gamma_{1}^{2} & \gamma_{2}^{2}
\end{array}\right) .
\end{aligned}
$$

Each constant, or free variable, corresponds to a symmetry. By setting a free variable equal to one and the rest equal to zero, the individual symmetries can be enumerated. Note that the number of free variables is the same for any number of agents ${ }^{1}$ that reference any number of neighbors, provided that if agent $\mathrm{A}$ references agent $\mathrm{B}$, then agent $\mathrm{B}$ references agent $\mathrm{A}$, i.e., the edges in the graph representing the system are undirected.

For brevity of presentation, we enumerate all the symmetries for a system with two agents:

- $X^{1}=\frac{\partial}{\partial t}$

- $X^{2}=\frac{\partial y}{\partial q^{1}}+\frac{\partial}{\partial q^{3}}$

- $X^{3}=\frac{\partial}{\partial q^{2}}+\frac{\partial}{\partial q^{4}}$

- $X^{4}=\exp (-t) \cdot\left(\frac{\partial}{\partial q^{1}}+\frac{\partial}{\partial q^{3}}\right)$

- $X^{5}=\exp (-t) \cdot\left(\frac{\partial}{\partial q^{2}}+\frac{\partial}{\partial q^{4}}\right)$

- $X^{6}=-q^{2} \frac{\partial}{\partial q^{1}}+q^{1} \frac{\partial}{\partial q^{2}}-q^{4} \frac{\partial}{\partial q^{3}}+q^{3} \frac{\partial}{\partial q^{4}}$

- $X^{7}=\left(q^{2}+q^{4}\right) \cdot\left(\frac{\partial}{\partial q^{1}}+\frac{\partial}{\partial q^{3}}\right)$

- $X^{8}=\left(q^{1}+q^{3}\right) \cdot\left(\frac{\partial}{\partial q^{2}}+\frac{\partial}{\partial q^{4}}\right)$

- $X^{9}=\left(q^{1}+q^{3}\right) \cdot\left(\frac{\partial}{\partial q^{1}}+\frac{\partial}{\partial q^{3}}\right)$

- $X^{10}=\left(q^{2}+q^{4}\right) \cdot\left(\frac{\partial}{\partial q^{2}}+\frac{\partial}{\partial q^{4}}\right)$.

${ }^{1}$ When there are three agents, an additional free variable is obtained in the $\bar{\Gamma}$ matrix, which becomes a circulant matrix. 
The first three symmetries have easy interpretations: translation in time, the $x$-direction and the $y$-direction, respectively. The fourth and fifth correspond to "center of mass coordinates" which decay in time due to the form of damping present in the example problem and the sixth is rigid body rotation.

\section{REDUCED-ORDER DYNAMICS}

For a given symmetry, $X^{k}=\xi_{k} \frac{\partial}{\partial t}+\eta_{k}^{i} \frac{\partial}{\partial q^{l}}$, it is possible to define a coordinate transformation such that $X^{k}=\frac{\partial}{\partial s}$, i.e., define one of the coordinates so that it is "parallel" with the symmetry. These new coordinates are in some sense canonical, and result in simplifying the expression for the dynamics of the system. This transformation is determined by defining $\left(r^{j}, s\right)$, such that $X^{k} s=1$ and $X^{k} r^{j}=0$. This may often be done by inspection, or in general by determining the trajectories (orbits) of the group generated by $X^{k}$. The trajectories are defined by

which can be rewritten as

$$
\frac{d q^{i}}{d \lambda}=\eta_{k}^{i}, \quad \frac{d t}{d \lambda}=\xi_{k}
$$

$$
\frac{d q^{1}}{\eta_{k}^{1}}=\frac{d q^{2}}{\eta_{k}^{2}}=\cdots=\frac{d q^{n}}{\eta_{k}^{n}}=\frac{d t}{\xi_{k}} .
$$

It is possible to take the initial values for these trajectories as $r^{j}$ and solve $X^{k} s=1$ by a line integral.

For the case of one equation, to solve $X r=0$, we take an orbit where $r$ equals a constant. This gives the expression

$$
d r=0=r_{, t} d t+r_{, q} d q
$$

Note that $X r=0$ can be rewritten as

$$
X r=\xi r_{, t}+\eta r_{, q}=0 .
$$

It is then possible to eliminate $r_{, t}$ and $r_{, q}$ by the prior two equations and obtain the expression

$$
\xi d q-\eta d t=0 \text {. }
$$

Now $r$ is the constant of integration that appears in the solution to the equation above. Recall that we defined for an orbit that $r$ is a constant. Each orbit of the system is defined by an $r$ value and it is possible to invert the system to obtain an expression for $r$ in terms of $q$ and $t$.

\section{EXAMPLE}

Returning to the example system

$$
\ddot{q}^{i}=-\dot{q}^{i}-\sum_{j \in \mathscr{N}_{i}}\left(q^{i}-q^{l}\right)\left(\left(q^{i}-q^{l}\right)^{2}+\left(q^{k}-q^{m}\right)^{2}-\hat{d}_{j}^{i}\right)
$$

(with $k, l$, and $m$ as defined previously) we will illustrate the reduction process for the two-agent system. Additionally, since the process requires multiple coordinate transformations, a subscript will be added to indicate the step in the process. In detail, for two-agents

$$
\begin{aligned}
& \left.\ddot{q}_{0}^{1}=-\dot{q}_{0}^{1}-\left(q_{0}^{1}-q_{0}^{3}\right)\left(q_{0}^{1}-q_{0}^{3}\right)^{2}+\left(q_{0}^{2}-q_{0}^{4}\right)^{2}-\hat{d}_{1}\right) \\
& \left.\ddot{q}_{0}^{2}=-\dot{q}_{0}^{2}-\left(q_{0}^{2}-q_{0}^{4}\right)\left(q_{0}^{1}-q_{0}^{3}\right)^{2}+\left(q_{0}^{2}-q_{0}^{4}\right)^{2}-\hat{d}_{1}\right) \\
& \left.\ddot{q}_{0}^{3}=-\dot{q}_{0}^{3}+\left(q_{0}^{1}-q_{0}^{3}\right)\left(q_{0}^{1}-q_{0}^{3}\right)^{2}+\left(q_{0}^{2}-q_{0}^{4}\right)^{2}-\hat{d}_{1}\right) \\
& \left.\ddot{q}_{0}^{4}=-\dot{q}_{0}^{4}+\left(q_{0}^{2}-q_{0}^{4}\right)\left(q_{0}^{1}-q_{0}^{3}\right)^{2}+\left(q_{0}^{2}-q_{0}^{4}\right)^{2}-\hat{d}_{1}\right) .
\end{aligned}
$$

\section{A. Translation in $x$-Direction}

Consider $X_{0}^{2}=\frac{\partial}{\partial q_{0}^{1}}+\frac{\partial}{\partial q_{0}^{3}}$. We need to find a coordinate transformation $\phi_{1}$ such that $X_{0}^{2} r^{j}=0$ and $X_{0}^{2} s^{1}=1$. One such transformation is

$$
\phi_{1}\left(q_{0}^{1}, q_{0}^{2}, q_{0}^{3}, q_{0}^{4}\right)=\left(q_{0}^{1}, q_{0}^{1}-q_{0}^{3}, q_{0}^{2}, q_{0}^{4}\right)=\left(s^{1}, r^{1}, r^{2}, r^{2}\right)
$$

Since this process will be repeated, the coordinates $\left(r^{1}, r^{2}, r^{3}\right)$ will be changed to $\left(q_{1}^{1}, q_{1}^{2}, q_{1}^{3}\right)$, respectively. The inverse transformation is

$$
\phi_{1}^{-1}\left(s^{1}, q_{1}^{1}, q_{1}^{2}, q_{2}^{3}\right)=\left(s^{1}, q_{1}^{2}, s^{1}-q_{1}^{1}, q_{1}^{3}\right)=\left(q_{0}^{1}, q_{0}^{2}, q_{0}^{3}, q_{0}^{4}\right)
$$

The updated symmetries are found by transforming the system to the new coordinates and recalculating the symmetries. The updated system of equations is

$$
\begin{aligned}
& \ddot{s}^{1}=-\dot{s}^{1}-q_{1}^{1}\left(\left(q_{1}^{1}\right)^{2}+\left(q_{1}^{2}-q_{1}^{3}\right)^{2}-\hat{d}_{1}\right) \\
& \ddot{q}_{1}^{1}=-\dot{q}_{1}^{1}-2 q_{1}^{1}\left(\left(q_{1}^{1}\right)^{2}+\left(q_{1}^{2}-q_{1}^{3}\right)^{2}-\hat{d}_{1}\right) \\
& \ddot{q}_{1}^{2}=-\dot{q}_{1}^{2}-\left(q_{1}^{2}-q_{1}^{3}\right)\left(\left(q_{1}^{1}\right)^{2}+\left(q_{1}^{2}-q_{1}^{3}\right)^{2}-\hat{d}_{1}\right) \\
& \ddot{q}_{1}^{3}=-\dot{q}_{1}^{3}+\left(q_{1}^{2}-q_{1}^{3}\right)\left(\left(q_{1}^{1}\right)^{2}+\left(q_{1}^{2}-q_{1}^{3}\right)^{2}-\hat{d}_{1}\right) .
\end{aligned}
$$

The most important point to note is that $s^{1}$ does not appear in the $q$-dynamics, i.e., the last three equations are independent of the first variable. We may consider the $q$ dynamics to be a form of a reduced system that may be solved independently. The $s^{1}$ equation can be solved if one wants to recover the full dynamics of the system, which in this case would be the absolute $x$ location and velocity.

Now, the process is repeated until all the symmetries are eliminated. The updated symmetries for the reduced $q$ dynamics are then $X_{1}^{1}=\frac{\partial}{\partial t}, X_{1}^{2}=0, X_{1}^{4}=0, X_{1}^{7}=0, X_{1}^{8}=0$, $X_{1}^{9}=0$ and

$$
\begin{aligned}
X_{1}^{3} & =\frac{\partial}{\partial q_{1}^{2}}+\frac{\partial}{\partial q_{1}^{3}} \\
X_{1}^{5} & =e^{-t} \cdot\left(\frac{\partial}{\partial q_{1}^{2}}+\frac{\partial}{\partial q_{1}^{3}}\right) \\
X_{1}^{6} & =-\left(q_{1}^{2}-q_{1}^{3}\right) \frac{\partial}{\partial q_{1}^{1}}+\frac{1}{2} q_{1}^{1}\left(\frac{\partial}{\partial q_{1}^{2}}-\frac{\partial}{\partial q_{1}^{3}}\right) \\
X_{1}^{10} & =\left(q_{1}^{2}+q_{1}^{3}\right) \cdot\left(\frac{\partial}{\partial q_{1}^{2}}+\frac{\partial}{\partial q_{1}^{3}}\right) .
\end{aligned}
$$

\section{B. Translation in y-Direction}

Now the process will be repeated for $X_{1}^{3}=\frac{\partial}{\partial q_{1}^{2}}+\frac{\partial}{\partial q_{1}^{3}}$. We need to find a coordinate transformation $\phi_{2}$ such that $X_{1}^{3} r^{j}=0$ and $X_{1}^{3} s^{2}=1$. Note that this is $s^{2}$ as $s^{1}$ will be left unchanged. One such transformation is

$$
\phi_{2}\left(q_{1}^{1}, q_{1}^{2}, q_{1}^{3}\right)=\left(q_{1}^{2}, q_{1}^{1}, q_{1}^{2}-q_{1}^{3}\right)=\left(s^{2}, r^{1}, r^{2}\right) .
$$

Again, the coordinates $\left(r^{1}, r^{2}\right)$ will be rewritten with $\left(q_{2}^{1}, q_{2}^{2}\right)$, respectively. The inverse transformation is

$$
\phi_{2}^{-1}\left(s^{2}, r^{1} r^{2}\right)=\left(q_{2}^{1}, s^{2}, s^{2}-q_{2}^{2}\right)=\left(q_{1}^{1}, q_{1}^{2}, q_{1}^{3}\right) .
$$


The transformation $\phi_{2}^{-1}$ is used to write the system in the new coordinates.

$$
\begin{aligned}
& \ddot{s}^{1}=-\dot{s}^{1}-q_{2}^{1}\left(\left(q_{2}^{1}\right)^{2}+\left(q_{2}^{2}\right)^{2}-\hat{d}_{1}\right) \\
& \ddot{s}^{2}=-\dot{s}^{2}-q_{2}^{2}\left(\left(q_{2}^{1}\right)^{2}+\left(q_{2}^{2}\right)^{2}-\hat{d}_{1}\right) \\
& \ddot{q}_{2}^{1}=-\dot{q}_{2}^{1}-2 q_{2}^{1}\left(\left(q_{2}^{1}\right)^{2}+\left(q_{2}^{2}\right)^{2}-\hat{d}_{1}\right) \\
& \ddot{q}_{2}^{1}=-\dot{q}_{2}^{2}-2 q_{2}^{2}\left(\left(q_{2}^{1}\right)^{2}+\left(q_{2}^{2}\right)^{2}-\hat{d}_{1}\right) .
\end{aligned}
$$

Using this additional symmetry has resulted in further decoupling of the equations of motion in the new coordinates. Specifically, the last two equations do not depend on the first two variables and may be solved independently. If the location or velocity in either the $x$ - or $y$-coordinates is needed, then the first two equations may be solved after solving the last two equations.

Now the updated symmetries for the reduced $q$-dynamics are then $X_{2}^{1}=\frac{\partial}{\partial t}, X_{2}^{2}=0, X_{2}^{3}=0, X_{2}^{4}=0, X_{2}^{5}=0, X_{2}^{7}=0$, $X_{2}^{8}=0 X_{2}^{9}=0, X_{2}^{10}=0$ and

$$
X_{2}^{6}=-q_{2}^{2} \frac{\partial}{\partial q_{2}^{1}}+q_{2}^{1} \frac{\partial}{\partial q_{2}^{2}} .
$$

\section{A Comment on the Coordinate Transformations Thus Far}

Depending on the application, it may be important that the agents are at rest (Case A), while other applications are only concerned that the agents are not moving relative to each other (Case B). This method is able to handle both cases. For Case B, each coordinate transformation thus far has been able to eliminate two variables, $s^{i}$ and $\dot{s}^{i}$. This is shown by the $q$ dynamics not being dependent on $s^{i}$ and $\dot{s}^{i}$. As will be shown with the rotational case, it is not always possible to eliminate two variables with one coordinate transformation, however it a pleasant result when it does occur. The reduced dynamics for this case is the $q$-dynamics

$$
\begin{aligned}
& \ddot{q}_{2}^{1}=-\dot{q}_{2}^{1}-2 q_{2}^{1}\left(\left(q_{2}^{1}\right)^{2}+\left(q_{2}^{2}\right)^{2}-\hat{d}_{1}\right) \\
& \ddot{q}_{2}^{2}=-\dot{q}_{2}^{2}-2 q_{2}^{2}\left(\left(q_{2}^{1}\right)^{2}+\left(q_{2}^{2}\right)^{2}-\hat{d}_{1}\right) .
\end{aligned}
$$

For Case A, each coordinate transformation has eliminated only one variable, which is all that the method guarantees. In this case is convenient to define a new variable $p^{i}=\dot{s}^{i}$, which defines the quadrature

$$
s^{i}=\int_{0}^{\infty} p^{i} d t
$$

The quadrature is now the equation to be solved if one wishes to determine where on the $x$ - and $y$-axis the formation is. The reduced dynamics is a system of two first order differential equations and two second order differential equations

$$
\begin{aligned}
& \dot{p}^{1}=-p^{1}-q_{2}^{1}\left(\left(q_{2}^{1}\right)^{2}+\left(q_{2}^{2}\right)^{2}-\hat{d}_{1}\right) \\
& \dot{p}^{2}=-p^{2}-q_{2}^{2}\left(\left(q_{2}^{1}\right)^{2}+\left(q_{2}^{2}\right)^{2}-\hat{d}_{1}\right) \\
& \ddot{q}_{2}^{1}=-\dot{q}_{2}^{1}-2 q_{2}^{1}\left(\left(q_{2}^{1}\right)^{2}+\left(q_{2}^{2}\right)^{2}-\hat{d}_{1}\right) \\
& \ddot{q}_{2}^{2}=-\dot{q}_{2}^{2}-2 q_{2}^{2}\left(\left(q_{2}^{1}\right)^{2}+\left(q_{2}^{2}\right)^{2}-\hat{d}_{1}\right) .
\end{aligned}
$$

Since the $q$ dynamics do not depend on the $p$ variables, it is possible solve the $q$ dynamics first and then solve the $p$ dynamics. For example, in terms of showing stability, one would proceed the same way as with Case B to show stability of the $q$ dynamics only, because that is specifically what is relevant to notions of "stability of the formation." It will be the focus of a future publication to work out the additional details regarding Lyapunov stability of the $q$ dynamics.

\section{Rotational Symmetry}

Now the rotational symmetry, $X_{2}^{6}$, will be used. For the prior two symmetries, it was convenient to keep the system as system of second order differential equations and define the coordinate transformation in terms of only the positions. This is not necessary to do so and at this point we convert the dynamics into a system of first order equations.

For both the original $x$ - and $y$-translation symmetries, the prolongation is equal to the original symmetry. This is due to the coefficient in front of the partials being a constant. The prolongation of the rotational symmetry is

$$
X_{2}^{6}=-q_{2}^{2} \frac{\partial}{\partial q_{2}^{1}}+q_{2}^{1} \frac{\partial}{\partial q_{2}^{2}}-\dot{q}_{2}^{2} \frac{\partial}{\partial \dot{q}_{2}^{1}}+\dot{q}_{2}^{1} \frac{\partial}{\partial \dot{q}_{2}^{2}} .
$$

To create a simplified set of reduced dynamics, a coordinate transformation will be defined such that the reduced dynamics are all first order differential equations. One such transformation is

$$
\begin{aligned}
& \phi_{3}\left(q_{2}^{1}, q_{2}^{2}, \dot{q}_{2}^{1}, \dot{q}_{2}^{2}\right)=\left[\begin{array}{c}
\arctan 2\left(\frac{q_{2}^{2}}{q_{2}^{1}}\right) \\
\left(q_{2}^{1}\right)^{2}+\left(q_{2}^{2}\right)^{2}-\hat{d}_{1} \\
\dot{q}_{2}^{1} q_{2}^{1}+\dot{q}_{2}^{2} q_{2}^{2} \\
\left(\dot{q}_{2}^{1}\right)^{2}+\left(\dot{q}_{2}^{2}\right)^{2}+\left(\left(q_{2}^{1}\right)^{2}+\left(q_{2}^{2}\right)^{2}-\hat{d}_{1}\right)^{2}
\end{array}\right] \\
& =\left[\begin{array}{llll}
s^{3} & r^{1} & r^{2} & r^{3}
\end{array}\right]^{T}
\end{aligned}
$$

with inverse in the configuration variables

$$
\phi_{3}^{-1}\left(s^{3}, r^{1}\right)=\left(\sqrt{r^{1}+\hat{d}_{1}} \cos s^{3}, \sqrt{r^{1}+\hat{d}_{1}} \sin s^{3}\right)=\left(q_{2}^{1}, q_{2}^{2}\right) \text {. }
$$

The transformation $\phi_{3}^{-1}$ is used to write the system in the new coordinates expressed as the four first-order equations

$$
\begin{aligned}
& \dot{s}^{3}=\sqrt{\left(r^{1}+\hat{d}_{1}\right)\left(r^{3}-\left(r^{1}\right)^{2}\right)-\left(r^{2}\right)^{2}} /\left(r^{1}+\hat{d}_{1}\right) \\
& \dot{r}^{1}=2 r^{2} \\
& \dot{r}^{2}=-2 \hat{d}_{1} r^{1}-r^{2}+r^{3}-3\left(r^{1}\right)^{2} \\
& \dot{r}^{3}=-2 r^{3}+2\left(r^{1}\right)^{2} .
\end{aligned}
$$

Note that $r^{1}$ is simply deviation from the desired distance, $r^{2}$ is related to the cross product between configuration and velocity, and hence is a type of relative angular momentum term, and $r^{3}$ is related to the kinetic energy. As was the case before, $s^{3}$ does not appear in any of the other equations. The $s^{3}$ equation only needs to be solved if one wishes to know the orientation of the formation. Thus, the rotational symmetry reduced the fourth-order system represented by the two, second-order $q_{2}$ equations in Equation 1, to the third-order system represented by the last three first-order equations in Equation 2.

As a check on the reduced equations, we compute a solution in the original coordinates and in the reduced, $r$ coordinates. Figures 3-4 illustrate solutions for a simulation with initial conditions given by $\left(\left(x_{1}, \dot{x}_{1}, y_{1}, \dot{y}_{1}, x_{2}, \dot{x}_{2}, y_{2}, \dot{y}_{2}\right)=\right.$ 


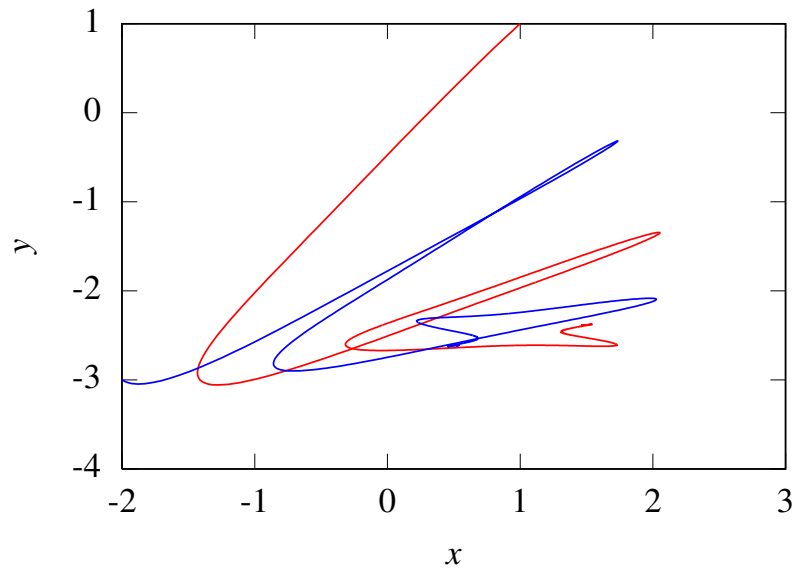

Fig. 3. Solution for two-agent system, agent 1 (red) and agent 2 (blue).

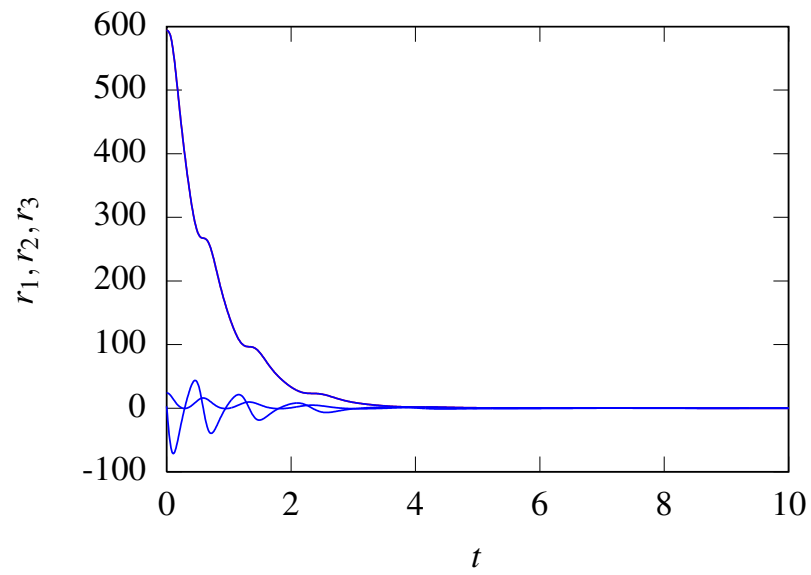

Fig. 4. Validation of reduced dynamics by comparing reduced coordinates computed from full system dynamics and reduced system dynamics.

$(1,0,1,0,-2,3,-3,-3$,$) . Figure 3$ illustrates the solution in the plane and Figure 4 illustrates the evolution of the three reduced $r$ coordinates. In Figure 4 the red curves, which can not be seen, are the values of the coordinates computed using the simulation data for the original (full) system transformed through the coordinate transformation; whereas, the blue curves (on top of the red ones) illustrate the simulation data from the reduced coordinates from Equation 2. For all three variables, $r_{1}$ through $r_{3}$ the curves for the two cases are identical, validating the relationship among the coordinate transformation and the original system dynamics and the reduced system dynamics in Equation 2.

Remark 1: Returning to one of the motivations for this work, it is clear that the origin is an equilibrium for the last three equations in 2 . Hence, the stability analysis for the formation control problem is reduced from an eightdimensional system (the original system with two masses, each with a second-order equation in both $x$ and $y$ with many feasible "formations" to a simple set of three equations with the origin as an equilibrium. Due to space limitations, completely fleshing out the application of this method to formation stability will be the subject of a future publication. The rest of this paper will consider the more general, $N$-agent system.

\section{USING THE SYMMETRIES FOR THE $N$-AGENT SYSTEM}

There are several different ways to extend these results to the general system. It is possible to pick $s^{1}=q_{0}^{1}$ again, or one can use an average of all of the odd coordinates. For simplicity and ease of notation, take $s^{1}=q_{0}^{1}$ and $\mathrm{s}^{2}=q_{0}^{2}$ where $s^{1}$ corresponds to $X_{0}^{2}$ and $s^{2}$ corresponds to $X_{0}^{3}$, where

$$
\begin{aligned}
& X_{0}^{2}=\frac{\partial}{\partial q_{0}^{1}}+\frac{\partial}{\partial q_{0}^{3}}+\cdots+\frac{\partial}{\partial q_{0}^{2 N-1}} \\
& X_{0}^{3}=\frac{\partial}{\partial q_{0}^{2}}+\frac{\partial}{\partial q_{0}^{4}}+\cdots+\frac{\partial}{\partial q_{0}^{2 N}} .
\end{aligned}
$$

Note that these two symmetries correspond to the $x-$ and $y-$ translation, respectively. Since these symmetries are independent, it is possible to define a coordinate transformation that will take care of both symmetries at once.

One possible coordinate transformation is

$$
\begin{array}{rlrl}
s^{1} & =q_{0}^{1} & s^{2} & =q_{0}^{2} \\
r^{1} & =q_{0}^{1}-q_{0}^{3} & r^{2} & =q_{0}^{2}-q_{0}^{4} \\
\vdots & \vdots & \\
r^{2 N-3} & =q_{0}^{1}-q_{0}^{2 N-1} & r^{2 N-2} & =q_{0}^{2}-q_{0}^{2 N} .
\end{array}
$$

As was done with the two coordinate system, $r^{i}$ will be rewritten as $q_{1}^{i}$. The inverse coordinate transformation is

$$
\begin{aligned}
q_{0}^{1} & =s^{1} & q_{0}^{2} & =s^{2} \\
q_{0}^{3} & =s^{1}-q_{1}^{1} & q_{0}^{4} & =s^{2}-q_{1}^{2} \\
\vdots & & \vdots & \\
q_{0}^{2 N-1} & =s^{1}-q_{1}^{2 N-3} & q_{0}^{2 N} & =s^{2}-q_{1}^{2 N-2} .
\end{aligned}
$$

The system of equations written in the new coordinates is now

$$
\begin{aligned}
\ddot{s}^{1} & =-\dot{s}^{1}-\sum_{j \in \mathscr{N}_{s}^{1}} d_{j}^{s^{1}}\left(q_{1}^{j}\right) \\
\ddot{s}^{2} & =-\dot{s}^{2}-\sum_{j \in \mathscr{N}_{s^{2}}} d_{j}^{s^{2}}\left(q_{1}^{j}\right) \\
\ddot{q}_{1}^{i} & =-\dot{q}^{i}+\sum_{s^{j} \in \mathscr{N}_{i}} d_{i}^{s^{j}}\left(q_{1}^{i}\right)-\sum_{j \in \mathscr{N}_{i}} d_{j}^{i}\left(q_{1}^{i}-q_{1}^{j}\right) \\
d_{j}^{s^{1}} & =\left(q_{1}^{j}\right)^{2}+\left(q^{j+1}\right)^{2}-\hat{d}_{j}^{s^{1}} \\
d_{j}^{s^{2}} & =\left(q_{1}^{j-1}\right)^{2}+\left(q_{1}^{j}\right)^{2}-\hat{d}_{j}^{s^{2}} \\
d_{j}^{i} & =\left(q^{i}-q^{l}\right)^{2}+\left(q^{k}-q^{m}\right)^{2}-\hat{d}_{j}^{i},
\end{aligned}
$$

where $i \in\{0, \ldots, 2 N-2\}, k=i-(-1)^{i}, l=i+2 j$ $(\bmod 2 N-2), m=k+2 j(\bmod 2 N-2)$, and care needs to be taken to make sure that $\mathscr{N}_{i}$ is defined properly to be consistent with the original system.

Note that the reduced system defined by $q_{1}^{i}$ does not depend on $s^{j}$, but rather only $\dot{s}^{j}$. This means that it is possible to define a quadrature

$$
s^{i}=\int_{0}^{\infty} p^{i} d t, \quad p^{i}=\dot{s}^{i} .
$$


To continue the reduction, it is not necessary to find all of the symmetries, but rather only the one to reduce the system by. The updated rotational symmetry is

$$
\begin{aligned}
X_{1}^{6}= & -p^{2} \frac{\partial}{\partial p^{1}}+p^{1} \frac{\partial}{\partial p^{2}}-q_{1}^{2} \frac{\partial}{\partial q_{1}^{1}}+q_{1}^{1} \frac{\partial}{\partial q_{1}^{2}} \\
& +\cdots+-q_{1}^{2 N-2} \frac{\partial}{\partial q_{1}^{2 N-3}}+q_{1}^{2 N-3} \frac{\partial}{\partial q_{1}^{2 N-2}} .
\end{aligned}
$$

From here one can choose to use $s^{3}=\arctan 2\left(q_{1}^{2} / q_{1}^{1}\right)$ again, or any other combination of the $p^{i}$,s and $q^{i}$ s that results in $X_{1}^{6} s^{3}=1$. A similar extension of the $r^{i}$,s for the two agent coordinate transformation can be used to define the coordinate transformation for the general case, however, due to the general case having an unspecified number of neighbors, it is not shown here.

It is important to note that these coordinate transformations are found based on the symmetries. Therefore, if another second order system of equations is found to have the symmetries $X_{0}^{2}, X_{0}^{3}$, and $X_{0}^{6}$, it is possible to use the same coordinate transformation to reduce the system.

\section{COnClusions And Future Work}

This paper computed all the symmetries for a planar multi-agent coordination control problem. This is important because many of the important attributes of the problem only depend on the relationship among the agents, and determining a formulation for the dynamics of the system that eliminates the absolution position and orientation of the formation helps reduce the complexity of the dynamics of the system to a reduced-dimension, and hence, simpler, representation. An important way in which this will be used is for formation stability analysis, which will be the subject of a future publication and is the focus of our current efforts.

\section{REFERENCES}

[1] P. J. Olver, Applications of Lie groups to differential equations. Springer, 2000, vol. 107.

[2] H. Stephani, Differential equations: their solution using symmetries. Cambridge University Press, 1989, malcolm MacCallum, Editor.

[3] J. E. Marsden and T. S. Ratiu, Introduction to mechanics and symmetry: a basic exposition of classical mechanical systems. Springer, 1999, vol. 17.

[4] A. M. Bloch, P. Krishnaprasad, J. E. Marsden, and R. M. Murray, "Nonholonomic mechanical systems with symmetry," Arch. Rational Mech. Anal., vol. 136, pp. 21-99, 1996.

[5] J. Koiller, "Reduction of some classical non-holonomic systems with symmetry," Archive for Rational Mechanics and Analysis, vol. 118, no. 2, pp. 113-148, 1992.

[6] J. E. Marsden, R. Montgomery, and T. S. Ratiu, "Reduction, symmetry and phases in mechanics," Memoirs of the Americal Mathematical Society, vol. 88, p. 436, 1990.

[7] J. E. Marsden and J. Scheurle, "Lagrangian reduction and the double spherical pendulum," ZAMP, vol. 44, pp. 17-43, 1993.

[8] — "The reduced euler-lagrange equations," Fields Institute Communications, vol. 1, pp. 139-164, 1993.

[9] H. Hanßmann, N. E. Leonard, and T. R. Smith, "Symmetry and reduction for coordinated rigid bodies," European journal of control, vol. 12, no. 2, pp. 176-194, 2006.

[10] J. Sztipanovits, X. Koutsoukos, G. Karsai, N. Kottenstette, P. Antsaklis, B. G. Vijay Gupta, J. Baras, and S. Wang, "Toward a science of cyberphysical system integration," Proceedings of the IEEE, 2011.
[11] J. Julliand, H. Mountassir, and E. Oudot, "Composability, compatibility, compositionality: automatic preservation of timed properties during incremental development," UFR Sciences et Techniques, Tech. Rep., 2007.

[12] B. Goodwine and P. Antsaklis, "Multi-agent compositional stability exploiting system symmetries," Automatica, vol. 49, no. 11, pp. 31583166, 2013.

[13] A. Jadbabaie, J. Lin, and A. S. Morse, "Coordination of groups of mobile autonomous agents using nearest neighbor rules," IEEE Transactions on Automatic Control, vol. 48, no. 6, pp. 988-1001, 2003.

[14] W. Ren, R. W. Beard, and E. M. Atkins, "Information consensus in multivehicle cooperative control," IEEE Control Systems Magazine, pp. 71-82, April 2007.

[15] J. A. Fax and R. M. Murray, "Information flow and cooperative control of vehicle formations," IEEE Transactions on Automatic Control, vol. 49, no. 9, pp. 1465- 1476, 2004.

[16] E. Rimon and D. E. Koditschek, "Exact robot navigation using artificial potential functions," IEEE Transactions on Robotics and Automation, vol. 8, no. 5, pp. 501-518, 1992.

[17] N. Leonard and E. Fiorelli, "Virtual leaders, artificial potentials, and coordinated cont," December 2001, pp. 2968-2973.

[18] R. Olfati-Saber and R. M. Murray, "Distributed cooperative control of multiple vehicle formations using structural potential functions," in Proceedings of the 2002 IFAC World Congress, July 2002.

[19] M. B. McMickell and B. Goodwine, "Reduction and nonlinear controllability of symmetric distributed systems," International Journal of Control, vol. 76, no. 18, pp. 1809-1822, 2003.

[20] _ - "Reduction and controllability of robotic systems with drift," in Proceedings of the 2002 IEEE International Conference on Robotics and Automation, May 2002.

[21] _ - "Motion planning for symmetric distributed robotic systems," in 2003 IEEE International Conference on Robotics and Automation, September 2003.

[22] _ - "Motion planning for nonlinear symmetric distributeed robotic systems," International Journal of Robotics Research, vol. 26, no. 10, pp. 1025-1041, October 2007.

[23] M. B. McMickell, B. Goodwine, and L. A. Montestruque, "Micabot: A robotic platform for large-scale distributed robotics," in 2003 IEEE International Conference on Robotics and Automation, September 2003.

[24] M. B. McMickell and B. Goodwine, "Reductionand controllability of symmetric distributed systems with robotic applications," in International Conference on Intelligent Robotics and Systems, vol. 3. IEEE/RSJ, October 2001, pp. 1232-1236.

[25] C. Belta and V. Kumar, "Abstraction and control for groups of robots," Robotics, IEEE Transactions on, vol. 20, no. 5, pp. 865-875, 2004.

[26] R. Olfati-Saber, "Flocking for multi-agent dynamic systems: Algorithms and theory," IEEE Transactions on Automatic Control, vol. 51, no. 3, pp. 401-420, 2006.

[27] R. Olfati-Saber and R. M. Murray, "Consensus problems in networks of agents with switching topology and time-delays," Automatic Control, IEEE Transactions on, vol. 49, no. 9, pp. 1520-1533, 2004.

[28] R. Olfati-Saber, J. A. Fax, and R. M. Murray, "Consensus and cooperation in networked multi-agent systems," Proceedings of the IEEE, vol. 95, no. 1, pp. 215-233, 2007.

[29] R. O. Saber and R. M. Murray, "Agreement problems in networks with directed graphs and switching topology," in Decision and Control, 2003. Proceedings. 42nd IEEE Conference on, vol. 4. IEEE, 2003, pp. 4126-4132. 